\title{
Laparoscopic Conversion to Loop Duodenojejunal Bypass with Sleeve Gastrectomy for Intractable Dumping Syndrome After Roux-en-Y Gastric Bypass-Two Case Reports
}

\author{
Chih-Kun Huang • Ming-Yu Wang • \\ Siddharth Sankar Das • Po-Chih Chang
}

Published online: 24 February 2015

(C) Springer Science+Business Media New York 2015

\begin{abstract}
Background Dumping syndrome is not infrequent after laparoscopic Roux-en-Y gastric bypass (LRYGB) and could result in dreaded complications, such as neuroglycopenia. For those refractory to diet modification or/and medication, regarded as intractable dumping syndrome, revision procedures should be taken into consideration. Herein, we make a video presentation of laparoscopic revision surgery for intractable dumping syndrome with unsatisfactory weight loss.

Methods Two diabetic, morbidly obese women (initial body mass index 36.6 and $41.4 \mathrm{~kg} / \mathrm{m}^{2}$ ) presented with intractable dumping syndrome 2 and 3 years after initial LRYGB, respectively. In addition, these patients had insufficient weight loss (body mass index 29 and $31 \mathrm{~kg} / \mathrm{m}^{2}$ ). Laparoscopic revision procedure of loop duodenojejunal bypass with sleeve gastrectomy was conducted to relieve their intractable conditions.

Results The mean operation time was 174 min (160 and 188), and the average blood loss was $60 \mathrm{~mL}$ (50 and 70). There was no intraoperative complication. Both patients had uneventful postoperative courses, and the average postoperative hospital stay was 2 days. The uncomfortable symptoms relieved successfully after the revision surgery. The Sigstad's score decreased to 2 points 6 months later, and the body mass index reduced to 26 and $28 \mathrm{~kg} / \mathrm{m}^{2}$.
\end{abstract}

Electronic supplementary material The online version of this article (doi:10.1007/s11695-015-1608-8) contains supplementary material, which is available to authorized users.

C.-K. Huang $\cdot$ S. S. Das $\cdot$ P.-C. Chang $(\bowtie)$

Bariatric and Metabolic International Surgery Center, E-Da hospital/

I-Shou University, Kaohsiung City, Taiwan

e-mail: dr.changpochih@hotmail.com

C.-K. Huang $\cdot$ M.-Y. Wang $\cdot$ P.-C. Chang

Division of General Surgery, Department of Surgery, E-Da hospital/

I-Shou University, Kaohsiung City, Taiwan
Conclusions Though long-term follow-up is warranted to draw a definite conclusion, loop duodenojejunal bypass with sleeve gastrectomy for pyloric restoration and malabsorptive effect remains an acceptable revision procedure to relive intractable dumping syndrome and successfully maintain sustained weight loss in our patients.

Keywords Dumping syndrome $\cdot$ Intractable dumping syndrome $\cdot$ Laparoscopic Roux-en-Y gastric bypass $\cdot$ Loop duodenojejunal bypass with sleeve gastrectomy

Conflict of Interest Chih-Kun Huang, Ming-Yu Wang, Siddharth Sankar Das, and Po-Chih Chang have no conflict of interest.

Funding There is no external source of funding.

Statement of Informed Consent Informed consent was obtained from all individual participants included in the study.

Statement of Human and Animal Rights All procedures performed in studies involving human participants were in accordance with the ethical standards of the institutional and/or national research committee and with the 1964 Helsinki declaration and its later amendments or comparable ethical standards. 